\title{
Análisis socio-ecológico de una iniciativa de restauración liderada por autoridades ambientales en Santander, Colombia
}

\author{
Socio-ecological analysis of a restoration initiative led \\ by environmental authorities in Santander, Colombia
}

\author{
Doris Duarte Hernández ${ }^{\circledR}$ y Andrés Avella Muñoz ${ }^{2}$
}

Duarte-H, D. y Avella-M, A. (2019). Análisis socio-ecológico de una iniciativa de restauración liderada por autoridades ambientales en Santander (Colombia). Colombia Forestal, 22(1), 68-86.

DOI: http://dx.doi.org/10.14483/2256201X.13101

Recepción: 7 de Marzo de 2018

\section{Resumen}

Se realizó el análisis socio-ecológico a un proyecto de restauración liderado por autoridades ambientales en Santander (Colombia); para ello se identificaron criterios sociales y ecológicos, se hizo una caracterización social y análisis multicriterio (con este último se obtuvo la valoración por criterio para cada actor y una valoración general del proyecto). A nivel social, la vinculación de actores fue muy buena (81/100), en contraste con la claridad conceptual y aporte de trabajo voluntario (50/100). A nivel ecológico, la prioridad para recuperar estructura, función y servicios ecosistémicos fue buena (79/100), mientras el número de especies autóctonas usadas fue regular (49/100). La gestión del proyecto fue buena (63/100), de acuerdo a los criterios socio-ecológicos definidos. Se concluyó que es importante planificar la fase de propagación de material vegetal, seleccionando especies según criterios funcionales e importancia para las comunidades que son actores fundamentales para la gobernanza y empoderamiento desde la formulación de los proyectos.

Palabras clave: análisis multicriterio, criterios, gobernanza, servicios ecosistémicos, socio-ecología.
Aprobación: 29 de octubre de 2018

\begin{abstract}
A socioecological analysis was carried out on a restoration project led by environmental authorities in Santander (Colombia). Social and ecological criteria were identified. A social characterization and a multi-criteria analysis were made, and the last one allowed obtaining the assessment by criterion for each stakeholder and a general project's assessment. At a social level, the linkage between actors was high (81/100), comparing to the conceptual clarity and contribution of voluntary work (50/100). At an ecological level, the priority to recover structure, function and ecosystem services was high (79/100), while the number of native species used was low (49/100). The project management had a good performance (63/100) according to the socio-ecological criteria. It was concluded that it is essential to plan the propagation phase of plant material, selecting species according to functional criteria and importance for the communities that are important actors for governance and empowerment under the project management.
\end{abstract}

Keywords: multi-criteria analysis, criteria, governance, ecosystem services, socioecology.

Universidad Distrital Francisco José de Caldas. Bogotá D.C., Colombia. doris.duarte.hernandez@gmail.com. Autor para correspondencia. 2 Instituto de Investigación de Recursos Biológicos Alexander von Humboldt. Bogotá D.C., Colombia. 


\section{INTRODUCCIÓN}

Ante un fenómeno de cambio global hacia la sostenibilidad, el desarrollo mundial actual vincula la dimensión ambiental y social (Jiménez, 2002). La problemática ecológica es posiblemente el mayor reto para la ciencia contemporánea, porque además de buscar enfoques que ofrezcan información confiable y completa, representa una amenaza para la humanidad (Toledo, Alarcón y Barón, 2002). En el pasado, los sistemas ecológicos y sociales eran objeto de análisis independiente; sin embargo, de acuerdo con Redman, Grove y Kuby (2004), su estudio aislado ya no es defendible pues las sociedades y su desarrollo influyen en la mayoría de sistemas ecológicos del planeta y esta condición a su vez retorna afectando los patrones de desarrollo (Meadows, Randers y Meadows, 2004). La socio-ecología surge de la articulación entre los aspectos sociales y ecológicos (Redman et al., 2004; Meadows et al., 2004), en esta las sociedades se consideran parte de los sistemas ecológicos de los que dependen y a los que aportan su experiencia (Von Glasersfeld, 1984; Jones, 2002). Estas son fundamentales en actividades como la restauración ecológica que es intencional y se adelanta para iniciar o acelerar la recuperación de un ecosistema, como una iniciativa que fomenta la recuperación sostenible, retornándolo (de ser posible) al estado previo a la alteración o a alguna de sus trayectorias sucesionales (SER, 2004; Clewell y Aronson, 2005).

El origen y consolidación de la restauración incluye un componente ecológico y uno social. Aun así, a lo largo de la historia se ha dado prioridad al ecológico y el social, de considerarse, solo se incluye cuando los proyectos se encuentran en ejecución, haciendo caso omiso a la percepción de las comunidades y poniendo en riesgo la continuidad de los procesos (Meadows et. al., 2004; Redman et.al., 2004; Zorrilla, 2007). La articulación de los aspectos sociales y ecológicos es indispensable para el desarrollo de proyectos de restauración y constituye el punto de partida para la generación de herramientas útiles en la formulación de nuevas estrategias y fortalecimiento de las existentes. En la mayoría de los casos, la restauración desarroIla muy bien sus aspectos técnicos, pero carece de evaluación social y lineamientos para apoyar las necesidades humanas (Balvanera et al., 2012); así, la gestión de iniciativas de restauración se convierte en la presentación de ambiciosas cifras para dar cumplimiento a metas regionales, nacionales o mundiales (Meli et al., 2016) y se realiza a partir de conceptos meramente ecológicos, dejando de lado la parte social que es necesaria en la construcción de procesos integrales para beneficio de los ecosistemas y las comunidades. De tal modo, esta investigación se plantea como necesaria para conocer el impacto real que generan los proyectos de restauración en los ecosistemas y las comunidades e identificar los aspectos técnicos, sociales, políticos, culturales y económicos que deben fortalecerse y mejorarse para asegurar el desarrollo y éxito de los procesos.

En Colombia, la Política Nacional para la Gestión de la Biodiversidad y sus Servicios Ecosistémicos (PNGIBSE) hace un reconocimiento de la biodiversidad como la base del bienestar y calidad de vida de los seres humanos. En este sentido, aunque no con la suficiente planificación y visión interdisciplinaria ecológica y social, el mayor impulsor de la restauración ha sido el Gobierno nacional a través del Ministerio de Ambiente y Desarrollo Sostenible (Mads) como gestor, financiador y ejecutor de proyectos en predios privados y públicos (Murcia y Guariguata, 2014), que en alianza con entidades como las corporaciones autónomas regionales (CAR) con frecuencia se vinculan al cumplimiento de metas de programas establecidos en los planes nacionales de desarrollo, contribuyendo a los desafíos mundiales trazados a la luz de la restauración ecológica (Murcia et al., 2016).

El estudio contempló la realización de un análisis socio-ecológico al proyecto de restauración liderado por autoridades ambientales (Mads y Corporación Autónoma Regional de Santander -CAS-), enmarcado en la meta nacional de restauración y establecido en Santander, Colombia. 
Incluyó la integración y evaluación de aspectos sociales y ecológicos relevantes del proyecto y se planteó para identificar los aspectos positivos y los que deben mejorarse como instrumentos claves para la gestión de nuevas iniciativas en la región, de acuerdo con los intereses de las instituciones gubernamentales, académicas y las comunidades.

\section{MATERIALES Y MÉTODOS}

\section{Área de estudio}

El proyecto de restauración se desarrolló en los municipios de Encino, Coromoro y Gámbita (Santander), localizados en el corredor de conservación Guantiva-La Rusia-Iguaque (figura 1), ubicado en la vertiente occidental de la cordillera Oriental, delimitado por la vertiente occidental del río Chicamocha, la oriental del río Suárez y ocupado por ecosistemas de bosques secos, subandinos, andinos, altoandinos y páramos (Sáenz-Jiménez, 2010).

La estrategia de restauración liderada por autoridades ambientales es una iniciativa nacional que tiene por objetivo el inicio de procesos de restauración a través de actividades de rehabilitación y recuperación con plantación de árboles y arbustos y construcción de aislamientos (MADS y CAS, 2012).

Se intervinieron 2845 ha: 2732 ha $(96 \%)$ en restauración espontánea y 113 ha $(4 \%)$ en restauración asistida, ubicadas en 30 predios privados (1148 ha) y 2 públicos (1697 ha). En algunos casos, varias unidades prediales pertenecen a un mismo propietario, por lo que al contabilizar el

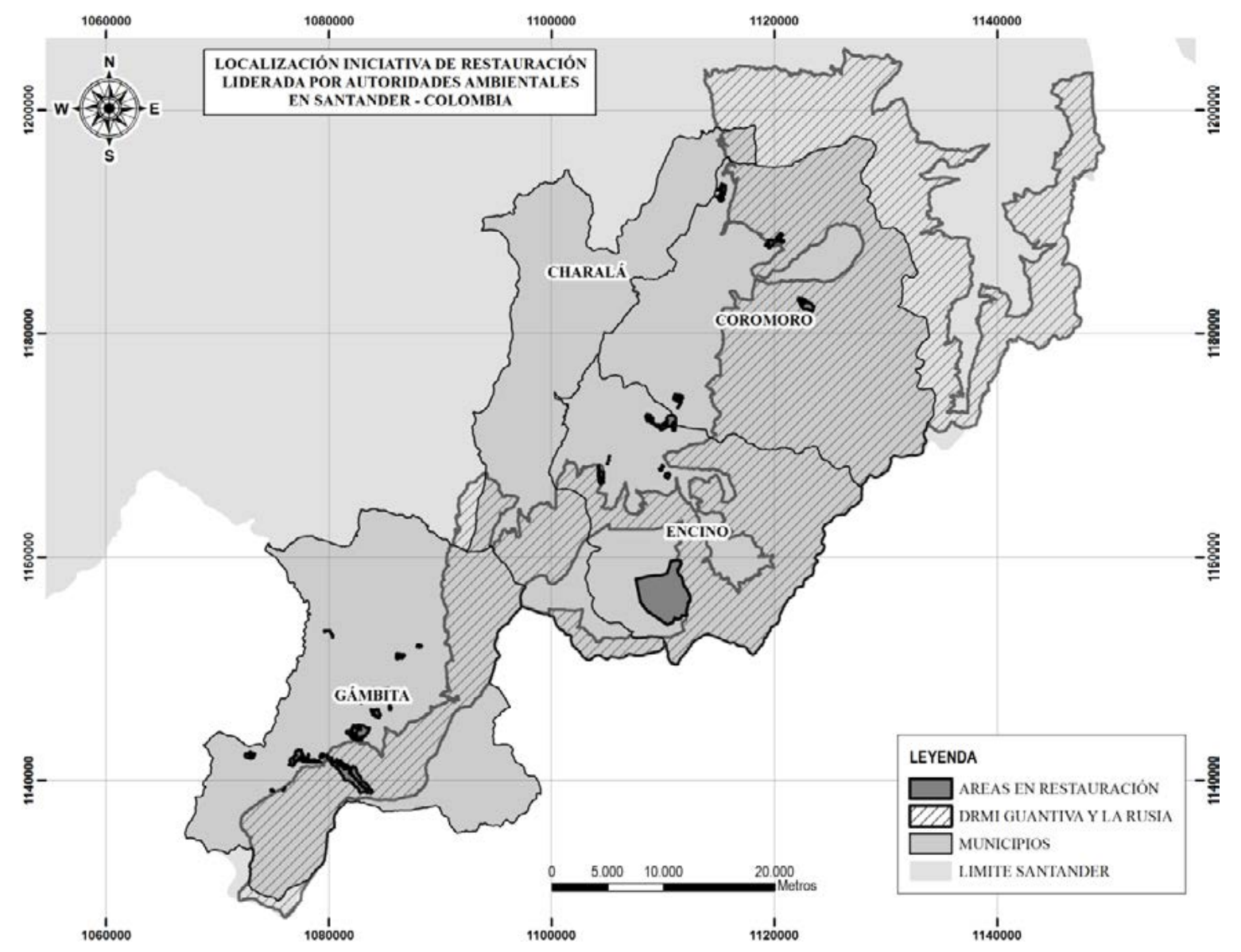

Figura 1. Localización de predios en los que se desarrolló el proyecto. 
total de propietarios asciende a 19. La restauración espontánea se desarrolló en áreas desprovistas de vegetación, con rastrojos bajos, medios y bosques aparentemente conservados; se establecieron cercas para favorecer la formación de cobertura vegetal y sucesión natural controlando agentes tensionantes como el sobrepastoreo, ramoneo y la cacería. Para reemplazar a futuro los postes deteriorados, en el perímetro de las cercas se plantaron 6 especies nativas y 4 introducidas. La restauración asistida se implementó en potreros, áreas desprovistas de vegetación o claros de bosque, con plantación de individuos vegetales de 16 especies nativas y 5 introducidas. La densidad fue de 800 plantas por hectárea distribuidas al azar (MADS y CAS, 2012).

\section{Métodos}

La investigación se desarrolló en tres etapas. En la primera se identificaron los criterios para la realización del análisis (anexo 1), siendo estos categorías amplias que permiten agrupar y organizar procesos similares o relacionados y posibilitan evaluar la efectividad de los proyectos en diferentes contextos, en un tiempo y espacio determinados (Basterra y Hess, 2004). Se consideran en conjunto para que en el tiempo reflejen la imagen completa del estado de un proyecto o su tendencia a la sostenibilidad (Wijewardana, Caswell y Palmberg-Lerche, 1997).

Para identificar los criterios, inicialmente se revisaron los 12 principios del enfoque ecosistémico (SCDB, 2004) y los atributos para determinar el logro de la restauración propuestos por la SER (2004); se tomaron los aplicables en una primera propuesta. Posteriormente, se revisaron recomendaciones para el desarrollo de proyectos de restauración hechas por Clewell y Aronson (2005), Vargas (2007), Zorrilla (2007), Orsi y Geneletti (2010) y Ribeiro-Pinto (2014) y se incluyeron las necesidades de las comunidades locales según lo propuesto por Max Neef, Elizalde y Hopenhayn (1998), Clewell y Aronson (2005), González, Montes, Rodríguez y Tapia (2008) y Orsi y Geneletti (2010).
La segunda etapa incluyó la caracterización y evaluación social con enfoque metodológico cualitativo. Esta se hizo a través de herramientas de desarrollo participativo (Geilfus, 1997) con la aplicación de entrevistas semiestructuradas y posterior análisis de datos de una muestra representativa que incluyó actores nacionales, regionales y locales (Díaz-Bravo, Torruco-García, Martínez-Hernández y Varela-Ruíz, 2013) y que fueron construidas a partir de los criterios socio-ecológicos identificados en la primera etapa.

Se realizaron 31 entrevistas semiestructuradas, 4 a profesionales del Ministerio de Ambiente y Desarrollo Sostenible y del Instituto de Investigaciones Biológicas Alexander von Humboldt, 9 a personal vinculado a la Gobernación de Santander, CAS, Santuario de Fauna y Flora Guanentá Alto Río Fonce, alcaldías municipales de Encino y Gámbita y 18 a los participantes comunitarios. Los actores nacionales fueron los encargados de la supervisión y apoyo para el fortalecimiento de capacidades del proyecto. Los regionales vinculados a la Secretaría de Agricultura Departamental encargados del seguimiento técnico por parte de la corporación, director del área protegida intervenida con restauración espontánea, alcaldes y funcionarios de apoyo de los municipios; y en locales, los participantes comunitarios de la iniciativa de restauración.

Las entrevistas a participantes comunitarios se realizaron en dos momentos y con diferentes instrumentos de recolección de información. El primero utilizando un formato guía similar al usado con los actores nacionales y regionales, y el segundo a través de conversaciones dirigidas por cinco preguntas orientadoras con las que se identificó información imperceptible en las primeras entrevistas. El formato guía de las entrevistas iniciales incluyó preguntas por cada uno de los criterios para todos los actores. Las preguntas en las conversaciones dirigidas con participantes comunitarios fueron: ¿cómo le ha parecido el proyecto?, ¿en la zona han existido iniciativas similares?, ¿ha tenido algún inconveniente?, ¿qué 
recomendaciones haría para mejorar?, ¿considera que el proyecto ha servido?

Con la información obtenida se hizo la evaluación a partir de la construcción de lineamientos para cuantificar las percepciones de los actores por criterio (tabla 1). Para cada criterio se evaluaron tres o cuatro lineamientos, estableciendo la relación porcentual entre el número de actores por percepción con respecto al número total de entrevistas por tipo de actor.

Tabla 1. Criterios y lineamientos para cuantificación de las percepciones.

\begin{tabular}{|c|c|}
\hline N. ${ }^{\circ}$ criterio & Lineamiento \\
\hline \multirow{4}{*}{ C1 } & 1. Conocimiento del proyecto de restauración \\
\hline & 2. Reciprocidad en intercambio de conocimientos \\
\hline & 3. Importancia de la participación de comunidades locales en la formulación de proyectos \\
\hline & 4. Participación en la formulación del proyecto \\
\hline \multirow{3}{*}{$\mathrm{C} 2$} & 1. Importancia de la vinculación \\
\hline & 2. Vinculación de diferentes actores \\
\hline & 3. Sensibilización como estrategia para vinculación a proyectos \\
\hline \multirow{3}{*}{ C3 } & 1. Claridad y facilidad de comunicación con diferentes actores \\
\hline & 2. Hay claridad pero se requiere continuidad \\
\hline & 3. Falta claridad \\
\hline \multirow{3}{*}{$\mathrm{C} 4$} & 1. Reducción de ingresos económicos por desarrollar proyectos de restauración \\
\hline & 2. Posibilidad de retribución por conservar \\
\hline & 3. Incremento del valor de los predios \\
\hline \multirow{3}{*}{ C5 } & 1. Conservación con capacitación y posibilidades de aprovechamiento sostenible \\
\hline & 2. Procesos de compensación, ordenamiento territorial, alternativas productivas y de mitigación \\
\hline & 3.Alternativas productivas agropecuarias sostenibles asociadas a la restauración \\
\hline \multirow{3}{*}{$\mathrm{C} 6$} & 1. Oportunidades de empleo masculino, femenino e ingresos adicionales \\
\hline & 2. Mejoramiento de predios \\
\hline & 3. Mejoramiento de ecosistemas para provisión de servicios ambientales \\
\hline \multirow{3}{*}{ C7 } & 1. Deterioro por cambio de uso de suelo y desarrollo de prácticas tradicionales \\
\hline & 2. Intención de cambio con capacitación y aprovechamiento sostenible \\
\hline & 3. Intención de conservar para asegurar disponibilidad de recurso hídrico \\
\hline \multirow{3}{*}{ C8 } & 1. Aporte de trabajo de comunidades porque es compromiso de todos \\
\hline & 2. Responsabilidad de comunidades para continuar el proyecto \\
\hline & 3. Necesidad de gestión para dar continuidad al proyecto \\
\hline \multirow{3}{*}{ C9 } & 1. Identificación de amenaza principal: degradación y deforestación por malas prácticas de uso \\
\hline & 2. Control con fortalecimiento de planificación predial \\
\hline & 3.Control con sensibilización ambiental y generación de proyectos productivos \\
\hline \multirow{3}{*}{$\mathrm{C} 10$} & 1. Énfasis en provisión de servicios ecosistémicos y mejoramiento de calidad de vida \\
\hline & 2. Mejoramiento de condiciones para asegurar disponibilidad de recurso hídrico \\
\hline & 3. Favorecimiento de conectividad para el hábitat y alimento de especies \\
\hline \multirow{3}{*}{ C11 } & 1. Áreas adecuadas según lineamientos del proyecto \\
\hline & 2. Áreas adecuadas, necesidad de ampliación a otras \\
\hline & 3. Áreas adecuadas, demoras en mantenimiento \\
\hline \multirow{3}{*}{$\mathrm{C} 12$} & 1. Adecuada selección de especies \\
\hline & 2. Inclusión de especies amenazadas o extintas localmente \\
\hline & 3. Especies nativas que aceleren procesos de restauración \\
\hline \multirow{3}{*}{$\mathrm{C} 13$} & 1. Responsabilidad de actores regionales, locales y academia \\
\hline & 2. Verificación de efectividad con visitas y parcelas de monitoreo \\
\hline & 3. Responsabilidad de todos los actores \\
\hline
\end{tabular}


En la tercera etapa se realizó un análisis multicriterio, considerado como una herramienta para la toma de decisiones en la que se pueden incluir varios criterios relacionados con aspectos cualitativos o cuantitativos y de forma multidimensional (Munda, 1993; Mendoza et al., 1999). Se analizaron los datos sociales a partir del enfoque de principios y criterios (Mendoza et al., 1999), en este caso socio-ecológicos. Los criterios identificados fueron evaluados con la caracterización y cuantificación de los lineamientos por cada actor y los resultados incorporados siguiendo el modelo de matriz distributiva multicriterio planteada por
Vargas-Isaza (2005). Para la estimación del valor de cada uno de los criterios se calculó el promedio generado de las percepciones por tipo de actor (tabla 2), posteriormente se estableció el promedio de las valoraciones de los actores en la matriz distributiva multicriterio (tabla 3). Del análisis multicriterio se obtuvo la valoración por criterio para cada actor y una valoración general del proyecto de restauración. Se estableció una escala de valoración relacionada con 5 categorías variables entre 0 y 100, así: deficiente (D): 0-19.9; insuficiente (I): 20-39.9; regular (R): 40-59.9; bueno (B): 60-79.9 y muy bueno $(\mathrm{MB})$ : 80-100.

Tabla 2. Evaluación de criterios socio-ecológicos según percepciones por tipo de actor.

\begin{tabular}{|c|c|c|c|c|c|c|c|c|}
\hline \multirow{5}{*}{\begin{tabular}{l}
\multicolumn{1}{c}{ Criterios } \\
C1. Participación en la \\
gestión de la restauración \\
e intercambio de saberes \\
con las comunidades
\end{tabular}} & \multirow{5}{*}{$\begin{array}{c}\text { Categoría } \\
\text { (R) }\end{array}$} & \multirow{5}{*}{$\begin{array}{l}\text { Lineamientos } \\
\text { Conocimiento del proyecto de restauración DR- } \\
\text { MI-GLR } \\
\text { Reciprocidad en intercambio de conocimientos } \\
\text { Importancia de la participación de comunidades } \\
\text { locales en la formulación de proyectos } \\
\text { Participación en la formulación del proyecto }\end{array}$} & \multicolumn{2}{|c|}{$\begin{array}{l}\text { Nacional } \\
(\%)\end{array}$} & \multicolumn{2}{|c|}{$\underset{(\%)}{\text { Regional }}$} & \multicolumn{2}{|c|}{$\begin{array}{c}\text { Local } \\
(\%)\end{array}$} \\
\hline & & & 50 & $\mathrm{R}$ & 100 & $\mathrm{MB}$ & 100 & $\mathrm{MB}$ \\
\hline & & & 75 & B & 78 & B & 100 & $\mathrm{MB}$ \\
\hline & & & 50 & $\mathrm{R}$ & 56 & $\mathrm{R}$ & 100 & $\mathrm{MB}$ \\
\hline & & & 0 & $\mathrm{D}$ & 0 & $\mathrm{D}$ & 0 & $\mathrm{D}$ \\
\hline & & Promedio & 44 & & 59 & & 75 & \\
\hline \multirow{4}{*}{$\begin{array}{l}\text { C2. Vinculación de } \\
\text { diversos actores: dueños } \\
\text { de tierra, estado, comuni- } \\
\text { dades }\end{array}$} & \multirow{4}{*}{$(\mathrm{MB})$} & Importancia de la participación & 100 & MB & 89 & $\mathrm{MB}$ & 100 & $\mathrm{MB}$ \\
\hline & & ación de difer & 75 & $\mathrm{~B}$ & 100 & $\mathrm{MB}$ & 100 & $\mathrm{MB}$ \\
\hline & & $\begin{array}{l}\text { Sensibilización como estrategia para vinculación } \\
\text { a proyectos }\end{array}$ & 50 & $\mathrm{R}$ & 44 & $\mathrm{R}$ & 67 & B \\
\hline & & Promedio & 75 & & 78 & & 89 & \\
\hline \multirow{4}{*}{$\begin{array}{l}\text { C3. Claridad conceptual y } \\
\text { metodológica utilizada en } \\
\text { el proyecto }\end{array}$} & \multirow{4}{*}{ (R) } & ilidad de comunicación con dife- & 50 & $\mathrm{R}$ & 78 & B & 89 & $\mathrm{MB}$ \\
\hline & & Hay claridad pero se requiere continuidad & - & $\mathrm{D}$ & 22 & I & 100 & $\mathrm{MB}$ \\
\hline & & Falt & 50 & $\mathrm{R}$ & 0 & $\mathrm{D}$ & 12 & $\mathrm{D}$ \\
\hline & & Pron & 50 & & 33 & & 67 & \\
\hline \multirow{4}{*}{$\begin{array}{l}\text { C4. Costo de uso del } \\
\text { suelo }\end{array}$} & \multirow{4}{*}{ (B) } & $\begin{array}{l}\text { Reducción de ingresos económicos por desarro- } \\
\text { llar proyectos de restauración }\end{array}$ & 25 & 1 & 22 & 1 & 17 & $\mathrm{D}$ \\
\hline & & Posibilidad de retribución por conservar & 100 & MB & 100 & $\mathrm{MB}$ & 100 & $\mathrm{MB}$ \\
\hline & & Incr & - & $\mathrm{D}$ & 78 & $\mathrm{~B}$ & 94 & $\mathrm{MB}$ \\
\hline & & Promedio & 63 & & 67 & & 70 & \\
\hline \multirow{4}{*}{$\begin{array}{l}\text { C5. Incorporación de } \\
\text { prácticas de conservación, } \\
\text { uso y aprovechamiento de } \\
\text { ecosistemas buscando el } \\
\text { equilibrio }\end{array}$} & \multirow{4}{*}{$(\mathrm{R})$} & $\begin{array}{l}\text { Conservación con capacitación y posibilidades de } \\
\text { aprovechamiento sostenible }\end{array}$ & 50 & $\mathrm{R}$ & 100 & $\mathrm{MB}$ & 61 & B \\
\hline & & $\begin{array}{l}\text { Procesos de compensación, ordenamiento territo- } \\
\text { rial, alternativas productivas y de mitigación }\end{array}$ & 25 & I & 44 & $\mathrm{R}$ & 89 & $M B$ \\
\hline & & $\begin{array}{l}\text { Alternativas productivas agropecuarias sostenibles } \\
\text { asociadas a la restauración }\end{array}$ & 50 & $\mathrm{R}$ & 44 & $\mathrm{R}$ & 50 & $\mathrm{R}$ \\
\hline & & Promedio & 42 & & 63 & & 67 & \\
\hline \multirow{3}{*}{$\begin{array}{l}\text { C6. Contribución al me- } \\
\text { joramiento de la calidad } \\
\text { de vida }\end{array}$} & \multirow{3}{*}{ (B) } & $\begin{array}{l}\text { Oportunidades de empleo masculino, femenino e } \\
\text { ingresos adicionales }\end{array}$ & 75 & B & 67 & B & 100 & $M B$ \\
\hline & & Mejoramiento de predios & 75 & B & 67 & B & 89 & $\mathrm{MB}$ \\
\hline & & $\begin{array}{l}\text { Mejoramiento de ecosistemas para provisión de } \\
\text { servicios ambientales }\end{array}$ & 75 & B & 33 & I & 89 & $\mathrm{MB}$ \\
\hline
\end{tabular}




\begin{tabular}{|c|c|c|c|c|c|c|c|c|}
\hline \multirow{5}{*}{\begin{tabular}{l}
\multicolumn{1}{c}{ Criterios } \\
C7. Intención común de \\
cambiar los patrones que \\
pueden estar influyendo \\
en el deterioro
\end{tabular}} & \multirow{5}{*}{$\begin{array}{c}\text { Categoría } \\
\\
\text { (B) }\end{array}$} & \multirow{5}{*}{$\begin{array}{l}\text { Lineamientos } \\
\text { Promedio } \\
\text { Deterioro por cambio de uso de suelo y desarrollo } \\
\text { de prácticas tradicionales } \\
\text { Intención de cambio con capacitación y aprove- } \\
\text { chamiento sostenible } \\
\text { Intención de conservar para asegurar disponibili- } \\
\text { dad de recurso hídrico }\end{array}$} & \multirow{2}{*}{\multicolumn{2}{|c|}{$\begin{array}{c}\begin{array}{c}\text { Nacional } \\
(\%)\end{array} \\
75 \\
\end{array}$}} & \multirow{2}{*}{\multicolumn{2}{|c|}{$\begin{array}{c}\text { Regional } \\
(\%)\end{array}$}} & \multicolumn{2}{|c|}{$\begin{array}{c}\text { Local } \\
(\%)\end{array}$} \\
\hline & & & & & & & 93 & \\
\hline & & & 100 & $\mathrm{MB}$ & 100 & $\mathrm{MB}$ & 89 & MB \\
\hline & & & 50 & R & 67 & B & 72 & B \\
\hline & & & 75 & B & 67 & B & 94 & $\mathrm{MB}$ \\
\hline & \multicolumn{2}{|r|}{ Promedio } & 75 & & \multicolumn{2}{|l|}{78} & \multicolumn{2}{|l|}{85} \\
\hline \multirow{4}{*}{$\begin{array}{l}\text { C8. Aporte de trabajo } \\
\text { voluntario }\end{array}$} & \multirow{4}{*}{ (R) } & $\begin{array}{l}\text { Aporte de trabajo de comunidades porque es } \\
\text { compromiso de todos }\end{array}$ & 50 & $\mathrm{R}$ & 22 & I & 50 & $\mathrm{R}$ \\
\hline & & $\begin{array}{l}\text { Responsabilidad de comunidades para continuar } \\
\text { el proyecto }\end{array}$ & 75 & B & 89 & $\mathrm{MB}$ & 50 & $\mathrm{R}$ \\
\hline & & $\begin{array}{l}\text { Necesidad de gestión para dar continuidad al } \\
\text { proyecto }\end{array}$ & 25 & 1 & - & $\mathrm{D}$ & 33 & 1 \\
\hline & & Promedio & 50 & & 56 & & 44 & \\
\hline \multirow{4}{*}{$\begin{array}{l}\text { C9. Control de amenazas } \\
\text { potenciales al ecosistema }\end{array}$} & \multirow{4}{*}{ (B) } & $\begin{array}{l}\text { Identificación de amenaza principal: degradación } \\
\text { y deforestación por malas prácticas de uso }\end{array}$ & 100 & $\mathrm{MB}$ & 100 & $\mathrm{MB}$ & 89 & MB \\
\hline & & $\begin{array}{l}\text { Control con fortalecimiento de planificación } \\
\text { predial }\end{array}$ & 50 & R & 22 & 1 & 11 & $\mathrm{D}$ \\
\hline & & $\begin{array}{l}\text { Control con sensibilización ambiental y genera- } \\
\text { ción de proyectos productivos }\end{array}$ & - & $\mathrm{D}$ & 100 & MB & 72 & B \\
\hline & & Promedio & 75 & & 74 & & 57 & \\
\hline \multirow{3}{*}{$\begin{array}{l}\text { C10. Prioridad en la } \\
\text { recuperación de la estruc- } \\
\text { tura, funcionamiento y } \\
\text { servicios ecosistémicos }\end{array}$} & \multirow{3}{*}{ (B) } & $\begin{array}{l}\text { Énfasis en provisión de servicios ecosistémicos y } \\
\text { mejoramiento de calidad de vida }\end{array}$ & 75 & B & 100 & MB & 100 & MB \\
\hline & & $\begin{array}{l}\text { Mejoramiento de condiciones para asegurar } \\
\text { disponibilidad de recurso hídrico }\end{array}$ & - & $\mathrm{D}$ & - & $\mathrm{D}$ & 94 & $\mathrm{MB}$ \\
\hline & & $\begin{array}{l}\text { Favorecimiento de conectividad para el hábitat y } \\
\text { alimento de especies }\end{array}$ & 25 & 1 & 78 & B & 100 & $\mathrm{MB}$ \\
\hline \multirow{4}{*}{$\begin{array}{l}\text { C11. Aplicación ade- } \\
\text { cuada y clara de escalas } \\
\text { temporales y espaciales } \\
\text { en el proyecto }\end{array}$} & \multirow{4}{*}{ (R) } & Promedio & 50 & & 89 & & 98 & \\
\hline & & Áreas adecuadas según lineamientos del proyecto & 50 & 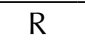 & 100 & MB & 100 & $\mathrm{MB}$ \\
\hline & & $\begin{array}{l}\text { Áreas adecuadas, necesidad de ampliación a } \\
\text { otras }\end{array}$ & 25 & 1 & 33 & 1 & 6 & D \\
\hline & & Áreas adecuadas, demoras en mantenimiento & 25 & I & 33 & 1 & 94 & $\mathrm{MB}$ \\
\hline \multirow{4}{*}{$\begin{array}{l}\text { C12. Número de especies } \\
\text { autóctonas o en peligro } \\
\text { de extinción presentes } \\
\text { para restauración }\end{array}$} & \multirow{4}{*}{ (R) } & Promedio & 33 & & 55 & & 67 & \\
\hline & & Adecuada selección de especies & 50 & $R$ & 88 & $\mathrm{MB}$ & 83 & $\mathrm{MB}$ \\
\hline & & $\begin{array}{l}\text { Inclusión de especies amenazadas o extintas } \\
\text { localmente }\end{array}$ & 25 & I & 67 & B & 100 & MB \\
\hline & & $\begin{array}{l}\text { Especies nativas que aceleren procesos de restau- } \\
\text { ración }\end{array}$ & 25 & 1 & 0 & $\mathrm{D}$ & 0 & $\mathrm{D}$ \\
\hline \multirow{5}{*}{$\begin{array}{l}\text { C13. Mecanismos de } \\
\text { monitoreo para evaluar el } \\
\text { proceso }\end{array}$} & \multirow{4}{*}{ (B) } & Promedio & 33 & & 52 & & 61 & \\
\hline & & $\begin{array}{l}\text { Responsabilidad de actores regionales, locales y } \\
\text { academia }\end{array}$ & 75 & B & 11 & $\mathrm{D}$ & 33 & 1 \\
\hline & & $\begin{array}{l}\text { Verificación de efectividad con visitas y parcelas } \\
\text { de monitoreo }\end{array}$ & 100 & $\mathrm{MB}$ & 77 & B & 100 & MB \\
\hline & & Responsabilidad de todos los actores & 25 & 1 & 67 & B & 50 & $\mathrm{R}$ \\
\hline & & Promedio & 67 & & 52 & & 61 & \\
\hline
\end{tabular}

Escala de valoración:

MB Muy bueno B Bueno R Regular I Insuficiente D Deficiente


Tabla 3. Matriz distributiva multicriterio

\begin{tabular}{|c|c|c|c|c|c|c|c|c|c|c|c|c|c|}
\hline \multirow{2}{*}{ Actores } & \multicolumn{13}{|c|}{ Criterios socio-ecológicos } \\
\hline & C-1 & $\mathrm{C}-2$ & C-3 & C-4 & C-5 & C-6 & C-7 & C-8 & C-9 & $\mathrm{C}-10$ & C-11 & $\mathrm{C}-12$ & C-13 \\
\hline Nacional & 44 & 75 & 50 & 63 & 42 & 75 & 75 & 50 & 75 & 50 & 33 & 33 & 67 \\
\hline Regional & 59 & 78 & 33 & 67 & 63 & 56 & 78 & 56 & 74 & 89 & 55 & 52 & 52 \\
\hline Local & 75 & 89 & 67 & 70 & 67 & 93 & 85 & 44 & 57 & 98 & 67 & 61 & 61 \\
\hline Promedio entrevistas & 59 & 81 & 50 & 67 & 57 & 74 & 79 & 50 & 69 & 79 & 52 & 49 & 60 \\
\hline Categoría entrevistas & $(\mathrm{R})$ & $(\mathrm{MB})$ & $(\mathrm{R})$ & (B) & $(\mathrm{R})$ & (B) & (B) & $(\mathrm{R})$ & (B) & (B) & $(\mathrm{R})$ & $(\mathrm{R})$ & (B) \\
\hline $\begin{array}{c}\text { Valoración del } \\
\text { proyecto }\end{array}$ & \multicolumn{13}{|c|}{63} \\
\hline Categoría del proyecto & \multicolumn{13}{|c|}{ Bueno (B) } \\
\hline
\end{tabular}

\section{RESULTADOS}

En el anexo 2 se incluyen las percepciones de los actores nacionales, regionales y locales, según los criterios identificados, lineamientos construidos y el papel que cada uno desempeñó en el proyecto. En la tabla 2 se presenta la evaluación de los criterios de acuerdo a las percepciones por tipo de actor según la escala de valoración definida que permite identificar los aspectos relevantes para cada actor desde el papel desempeñado en el proyecto. El criterio relacionado con la vinculación obtiene la mejor valoración porque los tres tipos de actores coinciden en afirmar que hubo posibilidad de formar parte de la ejecución del proyecto; en contraste, 6 de los 13 criterios definidos fueron valorados como buenos, lo que refleja un manejo adecuado de algunos aspectos socio-ecológicos y a diferencia de los anteriores, los restantes seis criterios fueron valorados como regulares, mostrando que hay aspectos fundamentales en el ejercicio de restauración que deben ser mejorados o fortalecidos para asegurar el éxito de los procesos.

En el análisis multicriterio la evaluación de percepciones de los criterios socio-ecológicos (tabla 3) indica que los mejores valorados fueron la vinculación de diferentes actores con 81, categorizado como muy bueno $(\mathrm{MB})$ y la prioridad en la recuperación de la estructura, funcionamiento y servicios ecosistémicos con 79 puntos, categorizado como bueno (B). En contraste, la claridad conceptual y metodológica utilizada en el proyecto, el aporte de trabajo voluntario y el número de especies autóctonas o en peligro de extinción usadas para restauración reflejan la valoración más baja con 50 puntos para los 2 primeros y 49 para el tercero, categorizada como regular (R).

La valoración del proyecto al evaluar los criterios socio-ecológicos definidos asciende a 63 puntos y se categoriza como bueno (B), indicando que la iniciativa de restauración tuvo en cuenta y aplicó algunos aspectos fundamentales.

\section{DISCUSIÓN}

A nivel social, la vinculación de diversos actores (dueños de tierra, Estado y comunidades) fue el aspecto más destacado. La integración del Mads, $\mathrm{IAvH}, \mathrm{CAS}$, Santuario de fauna y flora Guanentá alto río Fonce, las alcaldías municipales, juntas de acueductos veredales, dueños y trabajadores de predios, niños que fueron capacitados $y$, de manera especial, mujeres que en algunos casos trabajaron en construcción de cercas y plantación de árboles, se convirtió en un aspecto crucial que posibilitó el empoderamiento y despertó interés por parte de quienes inicialmente no integraron el proyecto. Esta situación puede ser previsible (SCDB, 2004; Vargas, 2007). 
En el análisis realizado al proyecto se enfatizó la importancia del género, resaltando el papel de la mujer al participar en actividades que generalmente son atribuidas a los hombres, además de las actividades domésticas que con frecuencia son menos valoradas socialmente al ser adoptadas como una relación de subordinación dentro de la comunidad (Rohlfs et al.,2000; CEPAL, 2006; Schkolnik, 2010). Se destaca que las acciones ejecutadas en el proyecto por las comunidades locales sirvieron como motivación para vincular a otros actores interesados en la restauración que no lo habían hecho antes porque habían perdido la credibilidad en las entidades estatales y en procesos que no han tenido continuidad en la región. A su vez, estas acciones también ofrecen oportunidades de empleo en labores diversas y generaron satisfacción al realizar un aporte a la conservación y restauración de ecosistemas que proveen múltiples servicios ambientales (De Young,1986; Miles, Sullivan y Kuo, 1998).

La intención común de cambiar los patrones que pueden influir en el deterioro fue un criterio relevante del proyecto. Se reconoció que las prácticas tradicionales generan daños irreversibles en los ecosistemas y amenazan la disponibilidad del recurso hídrico, uno de los aspectos que produce mayor preocupación a los actores locales pues reconocen la gradual disminución de caudales que tradicionalmente usaban en labores agropecuarias y domésticas. Para contrarrestar los daños, es fundamental buscar alternativas como la educación (Nilsson y Aradóttir, 2013) y el desarrollo de estrategias que faciliten el aprovechamiento sostenible acorde con el Plan Nacional de Restauración (MADS, 2015).

La contribución al mejoramiento de la calidad de vida es otro de los criterios que tiene buena percepción de los actores, al reconocer que el proyecto fue una oportunidad de empleo y que hubo mejoramiento de los predios y ecosistemas para la provisión de servicios ambientales que benefician a las comunidades, coincidiendo con las apreciaciones de Mace, Norris y Fitter (2012), MADS (2015) y Alexander, Aronson, Whaley, y
Lamb (2016). En el caso del proyecto, las comunidades reconocen que puede ser una alternativa y oportunidad para acceder al esquema de pago por servicios ecosistémicos, fortalecer las intenciones de mejorar y recuperar los ecosistemas y contribuir en la subsistencia, educación, participación e identidad de las comunidades; esto ratifica lo planteado por De Young (1986), Max Neef et al. (1998) y Wunder (2015). En este contexto Kolinjivadi y Sunderland, (2012) y Rodríguez y Merino (2016) han coincidido en señalar que las oportunidades para mejorar la subsistencia y diversificar las actividades productivas de las familias garantiza la sostenibilidad de los procesos y hogares a futuro. El mejoramiento de las condiciones de los actores locales también es una oportunidad para que las iniciativas de restauración trasciendan más allá del cumplimiento de metas y se conviertan en procesos liderados por las comunidades y escenarios para la investigación.

Otro criterio que contó con buena percepción fue el costo de uso del suelo, ya que los propietarios de predios consideraron que al desarrollar el proyecto hubo una valorización comercial y ambiental por las mejoras realizadas y el aporte a la conservación y recuperación de áreas estratégicas; contrario a lo señalado por Orsi y Geneletti (2010), manifestaron que al vincular áreas dedicadas a la conservación no dejaron de recibir recursos económicos. Es posible que la percepción de los actores esté ligada a la existencia de áreas alternas de explotación agropecuaria que facilitan el uso de otras áreas para el proyecto sin alterar sus principales actividades económicas, como también lo han expuesto Kelvin et al. (2014) en un estudio realizado para restaurar humedales en el Reino Unido.

La valoración más baja fue para el aporte de trabajo voluntario, el cual estuvo marcado por las diferencias en las percepciones de los actores. Los nacionales y regionales consideraron responsables a las comunidades, mientras que los locales están dispuestos a aportar su trabajo, siempre que haya financiación para dar continuidad a los proyectos 
pues dependen de estos recursos económicos para su subsistencia y al dedicarse solo a trabajar para mantener las iniciativas de restauración sin ninguna retribución económica se vería afectado el sustento mínimo para sus familias. En este caso deberían fomentarse los incentivos, la reducción de impuestos y pagos por servicios ecosistémicos diseñados para compensar comunidades comprometidas con la conservación como también lo han sugerido Jack, Kousky y Sims (2008) y Farley et al. (2010). Con respecto a la claridad conceptual y metodológica utilizada en el proyecto, a pesar de que hubo fluidez en los conceptos, como lo recomiendan Miles, Sullivan y Kuo (1998) y Clewell y Aronson (2005; 2007), es importante reforzar las actividades de capacitación y transmitir mensajes claros a las comunidades para evitar malos entendidos y facilitar la vinculación de más personas a las iniciativas de restauración.

La incorporación de prácticas de conservación, uso y aprovechamiento de ecosistemas buscando el equilibrio debe iniciar con actividades educativas que incentiven la utilización de experiencias encaminadas a la conservación, el uso sostenible y luego con el desarrollo de proyectos que ofrezcan alternativas productivas para las comunidades; que como lo afirma el World Bank (2010) dependen de los recursos naturales renovables y del capital natural para sus necesidades básicas y supervivencia. También es importante proyectar acciones y generar posibilidades para apoyar el uso sostenible articulado a procesos de restauración que fortalezcan una estrategia integral (Alexander et al., 2016).

Con respecto a la participación en la gestión de la restauración e intercambio de saberes, aunque se reconoció la importancia de las comunidades en la gestión y hubo intercambio de conocimientos, estas solo se vincularon en la ejecución del proyecto, pero no en su formulación, lo que generó un vacío al no tener en cuenta sus necesidades e intereses particulares, como también lo ha citado Bullock, Aronson, Newton, Pywell y Rey-Benayas (2011). En el análisis realizado Ilama la atención que el criterio mejor valorado fue la vinculación de diversos actores, pero esto sucedió cuando el proyecto se encontraba en ejecución porque en la formulación solamente participaron las autoridades ambientales, las cuales lo enfocaron de acuerdo a sus criterios y no según las necesidades de los ecosistemas e intereses de las comunidades que ocupan los territorios. Al tener en cuenta estos actores desde la formulación, se habría avanzado con mayor celeridad en la identificación de áreas que revisten importancia en términos de conservación y recuperación de ecosistemas, especies que existieron, han reducido sus poblaciones e interesa recuperar, zonas de recarga hídrica de interés común, principales agentes tensionantes, riesgos a los que está expuesto el proyecto y, en general, se habrían conocido los intereses y necesidades prioritarias de las comunidades.

La prioridad en la recuperación de la estructura, funcionamiento y servicios ecosistémicos fue un criterio bien valorado porque existe un interés particular en la recuperación de áreas boscosas que en otrora conservaban características para el hábitat de la fauna, provisión de productos forestales maderables y no maderables y zonas de recarga hídrica que mantienen constantemente el flujo de agua para uso doméstico y agropecuario. Esto ratifica la fuerte relación existente entre los servicios ecosistémicos y la restauración también sugerida por Alexander et al. (2016) que, aunque en ocasiones muestra resultados solo a mediano o largo plazo, garantiza mayor productividad en los ecosistemas restaurados que en los degradados (Mills et al., 2015) y favorece la recuperación de servicios ecosistémicos como el agua, fijación de CO2, suelo, biodiversidad, control de erosión y a su vez contribuye en el mejoramiento de la calidad de vida de los pobladores a largo plazo (Vargas, 2011; Cabrera y Ramírez, 2014).

El criterio referente a los mecanismos de monitoreo para evaluar el proceso fue otro categorizado como bueno (B), porque los actores evidenciaron que en el proyecto se establecieron parcelas permanentes y se llevó el registro periódico anual de variables dendrométricas, de sanidad y 
supervivencia; esto se ratifica en el hecho de que ocurrió un incendio que afectó 53 hectáreas de las 113 implementadas en restauración asistida y 8 de las 2732 en restauración espontánea y se establecieron 3 nuevas parcelas para evaluar la recuperación del área afectada e incremento de la cobertura vegetal. Sin embargo, se destaca que en el programa de monitoreo no se vinculó a la academia, pues no hay certeza de su continuidad al culminar el convenio MADS-CAS. Llama la atención que los actores nacionales delegan la responsabilidad en los regionales y locales, cuando deberían inculcar la investigación continua como lo sugieren Mills et al. (2015), utilizar aciertos y desaciertos para la gestión de nuevas iniciativas y no manejar la restauración como una receta.

Los criterios evaluados como regulares fueron el control de amenazas potenciales al ecosistema y la aplicación adecuada y clara de escalas temporales y espaciales en el proyecto. El primero porque pese a construir aislamientos para controlar las tensiones, el ganado sigue ingresando a los predios en proceso de restauración; los vecinos no ejercen control y con frecuencia ejecutan prácticas insostenibles poniendo en riesgo la integridad y progresiva recuperación del ecosistema como también lo han citado SER (2004), Vargas (2007) y González et al. (2008). Esto lleva a concluir que además de las actividades físicas, es fundamental crear empoderamiento y gobernanza de las comunidades para que los proyectos no permanezcan únicamente mientras hay aportes estatales $y$, por el contrario, perduren en el tiempo cumpliendo con la función para la que han sido formulados e implementados. El empoderamiento y la gobernanza de acuerdo a Nilsson y Aradóttir (2013) no pueden estar apartados de un proceso fuerte de educación ambiental en el cual se vinculen todos los integrantes de las comunidades.

Respecto a la aplicación adecuada de escalas temporales y espaciales, aunque las áreas fueron apropiadas según la necesidad de restauración, en el desarrollo de estas iniciativas es importante realizar un diagnóstico a escala de paisaje para identificar los fragmentos boscosos existentes y diseñar herramientas que favorezcan la conectividad. De igual manera, es necesario incluir otras áreas y establecer un plan de restauración en el que se proyecten los momentos claves para el mantenimiento y seguimiento, así como un mecanismo de control que evite el retraso de los procesos de restauración, teniendo en cuenta que las escalas temporales y espaciales deben definirse ecológicamente de acuerdo a la capacidad de recuperación del ecosistema, factores ecológicos, sociales y económicos, en áreas con necesidad de restauración, en periodos de mínimo cinco años y con monitoreo constante según Callicott (2002), Vargas (2007) y el MADS (2015) en el Plan Nacional de Restauración.

El criterio "número de especies autóctonas o en peligro de extinción usadas para restauración" tuvo la menor valoración debido a que la utilización de especies fue de acuerdo a la disponibilidad de material vegetal en viveros de la región y no como lo señalan Romero-Mejía (2005) y Vargas, Díaz-Triana, Reyes-Bejarano y Gómez-Ruíz (2012) a partir de estudios en los que se identifiquen sus rasgos de historia de vida, morfología, adaptación a las condiciones microambientales, utilidad para restaurar, preferencia por parte de las comunidades y presencia en las trayectorias sucesionales o ecosistema de referencia. Tampoco se tuvo en cuenta la utilización de especies de maderas finas que han reducido sus poblaciones o ya no existen, estas fueron sugeridas por los participantes de la iniciativa de restauración y pudieron producirse en viveros con una adecuada fase de propagación y según las necesidades del proyecto y de las comunidades.

Uno de los aspectos cruciales en procesos de restauración es el número de especies autóctonas o en peligro de extinción usadas. No obstante, para el proyecto fue de tan solo 16 especies nativas y 5 introducidas, contrario a la experiencia de restauración comunitaria desarrollada por Avella, Camacho y Torres (2016) para ecosistemas ubicados en la cuenca del río Guacha en Encino (Santander) 
perteneciente a la misma región en que se desarroIló el proyecto analizado, en la que se utilizaron más de 50 especies nativas. Esto sucedió porque al momento de la selección hubo necesidad de adaptarse a la disponibilidad de los viveros de la región, comprometiendo el éxito del proceso de restauración al no tener en cuenta las especies presentes en las trayectorias sucesionales y las que según sus atributos fueran útiles en los sitios a restaurar.

Con el análisis realizado, como generalidades cabe señalar que Colombia, al igual que otros países, está vinculada a los desafíos internacionales y para 2018 debe tener 300000 ha en proceso de restauración (DNP, 2014). Pero llama la atención que aún con estas ambiciosas metas no hay una definición clara de financiación ni fortalecimiento de la gobernanza local que asegure su permanencia en el tiempo y el cumplimiento de los objetivos de recuperación de ecosistemas. Por ejemplo, el proyecto estudiado se enmarcó en la meta nacional de 2014, como parte de la estrategia del gobierno para cumplir los compromisos del país a nivel mundial; pero, una vez realizado el análisis, se constata lo señalado por Balvanera et al. (2012) y Meli et al. (2016) para Latinoamérica con respecto a que la restauración ha surgido para mejorar los ecosistemas y el desarrollo socioeconómico, con una adecuada implementación de sus aspectos técnicos, pero con falencias en la evaluación y generación de opciones para satisfacer las necesidades humanas. Aunque en el caso del proyecto de restauración ecológica en Santander incluso algunos aspectos técnicos deben mejorarse para evitar que se sume al grupo de iniciativas que en Colombia contribuyen al cumplimiento de metas que con el pasar del tiempo no son sostenibles sin la debida financiación estatal.

Como herramienta para mejorar la gestión de iniciativas de restauración ecológica en la región, cada nuevo proyecto debe mantener la claridad en los aspectos técnicos. Del mismo modo, a las Comunidades, instituciones gubernamentales y académicas les corresponde contribuir y fortalecer la definición de los objetivos, la ubicación de áreas, el diseño, establecimiento, mantenimiento y monitoreo de las estrategias de restauración para asegurar el desarrollo y éxito de los procesos y convertirlos en una oportunidad de empleo, gobernanza y empoderamiento que ayude a mejorar el territorio $y$ la calidad de vida no solo con trabajos temporales, sino a través de alternativas que permitan el manejo, uso y aprovechamiento sostenible de la biodiversidad y sus servicios ecosistémicos.

\section{CONCLUSIONES}

El análisis socio-ecológico permitió a través de los criterios definidos evaluar como buena (63/100) la iniciativa de restauración e identificar que, a pesar de la valoración, se deben mejorar diversos aspectos para fortalecerla y asegurar su apropiación por parte de las comunidades como un mecanismo de gobernanza local y empoderamiento.

La evaluación de la iniciativa refleja la importancia de incluir aspectos técnicos, ecológicos y sociales, y del mismo modo, desarrollar acciones entre diferentes actores para la formulación, implementación y monitoreo de la restauración como una estrategia para que los proyectos regionales contribuyan a los compromisos que tiene el país con diferentes desafíos internacionales. Lo anterior para evitar que se conviertan en acciones mecánicas con las que se cubren áreas considerables solamente para cumplir metas; sin importar la gobernanza local a través de procesos perdurables.

La gestión de nuevas iniciativas de restauración ecológica en la región debe estar fundamentada en aspectos técnicos y sociales claros en los que las comunidades, instituciones gubernamentales y académicas y demás actores que interactúan en el territorio sean parte fundamental y contribuyan en la definición de los objetivos, ubicación de áreas, diseño, establecimiento, mantenimiento y monitoreo de estrategias para garantizar el fortalecimiento de los procesos y asegurar su éxito. 


\section{AGRADECIMIENTOS}

A Mónica Ramírez Guerrero por sus aportes en el análisis social. Al programa de Estímulos del Jardín Botánico de Bogotá. Al instituto ELTI de la Universidad de Yale. A Wilson Ramírez Hernández y Luis Mario Cárdenas Camacho por su revisión y aportes. A los profesionales del MADS, IAvH, Secretaría de Agricultura de Santander, CAS, alcaldías de Encino, Coromoro y Gámbita, Santuario de Flora y Fauna Guanentá Alto Río Fonce. De manera especial agradecemos a los participantes de la iniciativa de restauración, quienes enriquecieron la investigación y generaron aportes que serán la base para la formulación de nuevos procesos de restauración en la región.

\section{CONFLICTO DE INTERESES}

Los autores declaran no tener conflicto de intereses.

\section{CONTRIBUCIÓN POR AUTOR}

Los autores son los únicos responsables de la obra en todos los aspectos que condujeron a la elaboración de su publicación.

\section{REFERENCIAS}

Alexander, S., Aronson, J., Whaley, O. y Lamb, D. (2016). The relationship between ecological restoration and the ecosystem services concept. Ecology and society, 21(1), 34. DOI: https://doi. org/10.5751/ES-08288-210134

Avella, A., Camacho, L. y Torres, S. (2016). Restoration of Oak Forests (Quercus humboldtii) in the Colombian Andes: A Case Study of Landscape-Scale Ecological Restoration Initiatives in the Guacha River Watershed. En Restoration of Boreal and Temperate Forests (pp. 429-444). EE. UU.: Taylor Francis Group.
Balvanera, P., Uriarte, M., Almeida-Leñero, L., Altesor, A., DeClerck, F., Gardner, T... y Vallejos, M. (2012). Ecosystem services research in Latin America: the state of the art. Ecosystem Services, 2, 56-70. DOI: https://doi.org/10.1016/j.ecoser.2012.09.006

Basterra, N. I. y Hess, A. (2004). Selección de criterios ambientales para un manejo forestal sustentable. Centro de Gestión Ambiental y Ecología, comunicaciones científicas y tecnológicas. Universidad Nacional del Nordeste de Argentina. Resumen T: 016. 4 p.

Bullock, J. M., Aronson, J., Newton, A. C., PyweII, R. F. y Rey-Benayas, J. M. (2011). Restoration of ecosystem services and biodiversity: conflicts and opportunities. Trends in Ecology \& Evolution, 26(10), 541-549. DOI: https://doi.org/10.1016/j. tree.2011.06.011

Cabrera, M. y Ramirez, W. (eds.) (2014). Restauración ecológica de los páramos de Colombia. Transformación y herramientas para su conservación. Bogotá, Colombia: Instituto de Investigación de Recursos Biológicos Alexander von Humboldt.

Callicott, J. B. (2002). Choosing appropriate temporal and spatial scales for ecological restoration. J. BiosCi, 27(4), 409-420. DOI: https://doi.org/10.1007/ BF02704969

Clewell,A.yAronson,J. (2005). Motivationfortherestoration of ecosystems. Conservation Biology, 20(2), 420-428. DOI: https://doi.org/10.1111/j.1523-1739.2006.00340.x

Clewell, A. y Aronson, J. (2007). Ecological restoration: Principles, values and structure of an emerging profession. Washington D.C.: Island Press.

Comisión Económica para América Latina y El Caribe (Cepal), Unidad Mujer y Desarrollo (2006). Guía de asistencia técnica para la producción y el uso de indicadores de género. Santiago de Chile, Chile: Cepal.

De Young, R. (1986). Some phychological aspects of recycling: the structure of conservation satisfactions. Environment and behavior, 18, 435-449. DOI: https://doi.org/10.1177/0013916586184001

Díaz-Bravo, L., Torruco-García, U., Martínez-Hernández, M. y Varela-Ruíz, M. (2013). La entrevista, recurso flexible y dinámico. Investigación en 
Educación Media, 2(7), 162-167. DOI: https://doi. org/10.1016/S2007-5057(13)72706-6

Departamento Nacional de Planeación (DNP) (2014). Bases del Plan Nacional de Desarrollo 20142018, Todos por un Nuevo País. Recuperado de DOI: https://colaboracion.dnp.gov.co/cdt/prensa/ bases $\% 20$ plan\%20nacional\%20de\%20desarroIlo\%202014-2018.pdf

Farley, J., Aquino, A., Daniels, A., Moulaert, A., Lee, D. y Krause, A. (2010). Global mechanisms for sustaining and enhancing PES schemes. Ecological economics, 69, 2075-2084. DOI: https://doi.org/10.1016/j.ecolecon.2010.02.016

Geilfus, F. (1997). 80 herramientas para el desarroIlo participativo: diagnóstico, planificación, monitoreo, evaluación. San Salvador, El Salvador: Prochalate-IICA.

González, J. A., Montes, C., Rodríguez, J. y Tapia, W. (2008). Rethinking the Galapagos Islands as a complex social-ecological system: implications for conservation and management. Ecology and Society, 13(2), 13. DOI: https://doi.org/10.5751/ ES-02557-130213

Jack, B. K., Kousky, C. y Sims, K. R. (2008). Designing payments for ecosystem services: lessons from previous experience with incentive-based mechanisms. Proceedings of the National Academy of Sciences of the United States of America, 105(28), 9465-9470. DOI: https://doi.org/10.1073/ pnas.0705503104

Jimenez-Herrero, L. M. (2002). La sostenibilidad como proceso de equilibrio dinámico y adaptación al cambio. Información Comercial Española: Revista de economía, 800, 65-84.

Jones, S. (2002). Social constructionism and the environment: through the quagmire. Global Environmental Change, 12, 247-251. DOI: https://doi. org/10.1016/S0959-3780(02)00062-6

Kangas, J. y Store, R. (2003). Internet and teledemocracy in participatory planning of natural resources management. Landscape and Urban Planning, 62, 89-101. DOI: https://doi.org/10.1016/ S0169-2046(02)00125-1
Kelvin, P., Balmford, A., Field, R., Lamb, A., Birch, J., Bradbury, R... y Hughes, F. (2014). Benefits and costs of ecological restoration: Rapid assessment of changing ecosystem service values at a U.K. wetland. Ecology and evolution, 4(20), 3875-3886. DOI: https://doi.org/10.1002/ece3.1248

Kolinjivadi, V. y Sunderland, T. (2012). A review of two payment schemes for watershed services from Chiba and Vietnam: the interface of government control and PES theory. Ecology and Society, 17(4), 10. DOI: https://doi.org/10.5751/ES-05057-170410

Mace, G. M., Norris, K. y Fitter, A. H. (2012). Biodiversity and ecosystem services: a multilayered relationship. Trends in Ecology \& Evolution, 27(1),19-26. DOI: https://doi.org/10.1016/j.tree.2011.08.006

Max Neef, M., Elizalde, A. y Hopenhayn, M. (1998). Desarrollo a escala humana, conceptos, aplicaciones y algunas reflexiones. Montevideo, Uruguay: Editorial Nordan, Icaria Editorial.

Meadows, D. H., Randers, J. y Meadows, D. (2004). Limits to growth: The 30-year update. Londres, Inglaterra: Chelsea Green Publishing Company.

Meli, P., Herrera, F., Melo, F., Pinto, S., Aguirre, N., Musálem, K., Minaverry, C., Ramírez, W. y Brancalion, P. (2016). Four approaches to guide ecological restoration in Latin America. Restoration Ecology, 25(2), 156-163. DOI: DOI: https://doi.org/10.1111/ rec. 12473

Mendoza, G. A., Macoun, P., Prabhu, R., Sukadri, D., Purnomo, H, Hartanto, H. (1999). Guidelines for applying Multi-Criteria Analysis to the Assessment of Criteria and Indicators. Yakarta, Indonesia: Center for International Forestry Research.

Miles, I., Sullivan, W. y Kuo, F. (1998). Ecological restoration volunteers: the benefits of participation. Urban ecosystems, 2, 27-41. DOI: https://doi. org/10.1023/A:1009501515335

Mills, A. J., Van der Vyver, M., Gordon, I. J., Patwardhan, A., Marais, C., Blignaut, J. y Kgope, B. (2015). Prescribing innovation within a large-scale restoration programme in degraded subtropical thicket in South Africa. Forests, 6(11), 4328-4348. DOI: https://doi.org/10.3390/f6114328 
Ministerio de Ambiente y Desarrollo Sostenible (MADS) y Corporación Autónoma Regional de Santander (CAS) (2012). Proyecto: inicio de procesos de restauración, a través de la rehabilitación y recuperación en el distrito regional de manejo integrado que comprende los páramos de Guantiva y La Rusia, bosques de roble y sus zonas aledañas, en el departamento de Santander -fase 1. San Gil, Santander: MADS, CAS.

Ministerio de Ambiente y Desarrollo Sostenible (MADS) (2015). Plan nacional de restauración. Restauración ecológica, rehabilitación y recuperación de áreas disturbadas. Bogotá, Colombia: Mads.

Munda, G. (1993). Fuzzy information in multicriteria environmental evaluation models. Ispra: Joint Research Center.

Murcia, C. y Guariguata, M. (2014). La restauración ecológica en Colombia: tendencias, necesidades y oportunidades. Bogor, Indonesia: Cifor.

Murcia, C., Guariguata, M., Andrade, A., Andrade, G.I., Aronson, J. Escobar, E. M., Etter, A., Moreno, F. H., Ramírez, W. y Montes, E. (2016). Challenges and Prospects for Scaling-up Ecological Restoration to Meet International Commitments: Colombia as a Case Study. Conservation Letters, 9(3), 213-220. DOI: https://doi.org/10.1111/conl.12199

Nilsson, C. y Aradóttir, A. (2013). Ecological and social aspects of ecological restoration: New challenges and opportunities for Northern Regions. Ecology and society, 18(4), 35. DOI: https://doi. org/10.5751/ES-06045-180435

Orsi, F. y Geneletti, D. (2010). Identifying priority areas for Forest Landscape Restoration in Chiapas (Mexico): An operational approach combining ecological and socioeconomic criteria. Landscape and Urban Planning, 94, 20-30. DOI: https://doi.org/10.1016/j.landurbplan.2009.07.014

Redman, C. H. L., Grove, J. M. y Kuby, L. H. (2004). Integrating social science into the Long-Term Ecological Research (LTER) Network: social dimensions of ecological change and ecological dimensions of social change. Ecosystems, 7, 161-171. DOI: https://doi.org/10.1007/s10021-003-0215-z
Ribeiro-Pinto, S. R. (2014). Protocolo de monitoreo para el pacto de restauración de la mata Atlántica. En B. Baptiste, Monitoreo de áreas en proceso de restauración ecológica. Curso de campo llevado a cabo en el Santuario de Flora y Fauna Otún Quimbaya, Risaralda, Colombia.

Rodríguez, K. J. y Merino, L. (2016). Contextualizing context in the analysis of payment for ecosystem services. Ecosystem services, 23(2017), 259-267.

Rohlfs, I., Borrel, C., Anitua, C., Artazcoz, L., Colomer, C., Escribá, V., García Calvente, M., Llacer, A., Mazarrasa, L., Pasarín, M. I., Peiró, R. y Valls Llobet, C. (2000). La importancia de la perspectiva de género en las encuestas de salud. Gac Sanit, 14(2), 146-155. DOI: https://doi.org/10.1016/S0213-9111(00)71448-8

Romero-Mejía, A. (2005). Propuesta metodológica para seleccionar especies pioneras leñosas con fines de restauración ecológica dentro de la reserva biológica Cachalú (Encino-Santander). Colombia Forestal, 9(18), 52-59. DOI: https://doi.org/10.14483/udistrital.jour.colomb.for.2005.1.a04

Sáenz-Jiménez, F. A. (2010). Aproximación a la fauna asociada a los bosques de roble del Corredor Guantiva-La Rusia-Iguaque (Boyacá, Santader, Colombia). Colombia Forestal, 13(2), 299-334. DOI: https://doi. org/10.14483/udistrital.jour.colomb.for.2010.2.a08

Schkolnik, S. (2010). Acerca de la inclusión del enfoque de género en los censos de población y vivienda. En S. Huenchuan y G. Bay (eds.), Notas de población N. ${ }^{\circ} 91$ (7-41). Santiago de Chile: Cepal.

Secretaría del Convenio Sobre la Diversidad Biológica (SCDB) (2004). Enfoque por ecosistemas. Montreal, Canadá: SCDB.

Society for Ecological Restoration International Science y Policy Working Group (SER) (2004). The SER International Primer on Ecological Restoration. Tucson, Arizona: SER.

Toledo, V., P. Alarcón. y L. Barón. (2002). Revisualizar lo rural: un enfoque socioecológico. Gaceta Ecológica, 62, 7-20.

Vargas-Isaza, O. (2005). La evaluación multicriterio social y su aporte a la conservación de los bosques. Revista Facultad Nacional de Agronomía, 58(1). 
Vargas, O. (ed.) (2007). Guía metodológica para la restauración ecológica del bosque altoandino. Bogotá, Colombia: Grupo de Restauración Ecológica, Departamento de Biología Universidad Nacional de Colombia.

Vargas, O. (2011). Restauración ecológica: biodiversidad y conservación. Acta Biológica Colombiana, 16(2), 221-246.

Vargas, O., Díaz-Triana, J., Reyes-Bejarano, S. y Gómez-Ruíz, P. (2012). Guías técnicas para la restauración ecológica de los ecosistemas de Colombia. Bogotá, Colombia: Ministerio de Ambiente y Desarrollo Sostenible, Universidad Nacional de Colombia.

Von Glasersfeld, E. (1984). An introduction to radical constructivism. En: P. Watzlawick (ed.), The invented reality (pp. 1-16). Nueva York: Norton.

Wijewardana, D., Caswell, S.J. y Palmberg-Lerche, C. (1997). Criterios e indicadores para la ordenación forestal sostenible. En La actividad forestal para un desarrollo sostenible: hacia el siglo XXI. Congreso Forestal Mundial, 11, Antalya (Turkey) (pp. 13-22).

World Bank (2010). The changing wealth of nations: measuring sustainable development in the new millennium. Washington, D.C.: World Bank. DOI: https://doi.org/10.1596/978-0-8213-8488-6

Wunder, S. (2015). Revising the concept of payments for environmental services. Ecologycal Economics, 117, 234-243. DOI: https://doi.org/10.1016/j. ecolecon.2014.08.016

Zorrilla, M. (2007). La influencia de los aspectos sociales sobre la alteración ambiental y la restauración ecológica. En O. Sánchez, E. Peters, R. Márquez, E. Vega, G. Portales, M. Valdés y D. Azuara (eds.), Temas sobre restauración ecológica (pp. 31-43). México D.F.: Instituto Nacional de Ecología. 
Anexo 1. Criterios socio-ecológicos definidos para el análisis.

\section{Criterios socio-ecológicos}

C1. Participación en la gestión de la restauración e intercambio de saberes con las comunidades. Los interesados directos a nivel local deben participar en la gestión, garantizando cooperación entre las partes, mayor responsabilidad, apropiación, integración de conocimientos e interés por el desarrollo y continuidad de los procesos (SCDB, 2004; Clewell y Aronson, 2005 y González et al., 2008).

C2. Vinculación de diversos actores: dueños de tierra, Estado, comunidades y ONG. Cada actor cumple un papel determinante en los procesos de restauración y, por existir diversos niveles de interés, es necesaria la interacción y coordinación de las decisiones, vinculando a todos los actores como estrategia para fortalecer los procesos. La SCDB (2004) en el enfoque ecosistémico relaciona este criterio, reconociendo al ser humano como componente esencial.

C3. Claridad conceptual y metodológica utilizada en el proyecto. Hace referencia a la importancia de transmitir mensajes claros y contundentes relacionados con los objetivos del proceso, el proyecto y su ejecución para asegurar la vinculación, participación activa y apoyo decidido y constante de los actores (Clewell y Aronson, 2005).

C4. Costo de uso del suelo. Es determinante en el desarrollo y éxito de proyectos y está relacionado con la valoración del suelo destinado a restauración ecológica. Incluye la evaluación de actividades económicas que se desarrollan en el área destinada a iniciativas de restauración y la cuantificación de los ingresos económicos que se dejan de percibir por el cambio de uso, o por el uso de suelos para actividades diferentes a las productivas, en el periodo de tiempo de ejecución de los proyectos (Orsi y Geneletti, 2010).

C5. Incorporación de prácticas de conservación, uso y aprovechamiento de ecosistemas buscando el equilibrio. La adecuada administración y uso de los recursos naturales direcciona a las comunidades con su identidad cultural a la apropiación del territorio, promoviendo la conservación y utilización sostenible de los ecosistemas, para asegurar su subsistencia y la persistencia de los productos y subproductos en el tiempo (Kangas y Store, 2003; SCDB, 2004; Vargas, 2007; Zorrilla, 2007; González et al., 2008).

C6. Contribución al mejoramiento de la calidad de vida. La gestión de proyectos de restauración ecológica debe garantizar el mejoramiento de las condiciones de vida de las comunidades con la inclusión de principios ecológicos y socioeconómicos que garanticen la generación de oportunidades e incentiven la capacidad de proteger la biodiversidad y funcionamiento de ecosistemas. Orsi y Geneletti (2010) recomiendan este criterio como una oportunidad para vincular las comunidades y mejorar sus condiciones. La calidad de vida está relacionada con las posibilidades de satisfacer las necesidades fundamentales que se interrelacionan e interactúan; las hay de ser, tener, hacer y estar y las de subsistencia, protección, afecto, entendimiento, participación, ocio, creación, identidad, libertad y educación, estas últimas entendidas como satisfactores de necesidades fundamentales de subsistencia y no como necesidades propiamente dichas (Max Neef et al., 1998).

C7. Intención común de cambiar los patrones que pueden estar influyendo en el deterioro. Es importante identificar las principales acciones que ocasionan la degradación de los ecosistemas y en caso de ser atribuibles a los sistemas productivos analizar si se pueden combinar con acciones de restauración que favorezcan la sostenibilidad. Zorrilla (2007) propone la integración de diferentes actores con influencia en el territorio, como mecanismo para conocer el estado actual de los ecosistemas, aportar en la consolidación de los procesos de apropiación y crear la necesidad de cambiar las prácticas tradicionales que ocasionan el daño e incrementan su deterioro.

C8. Aporte de trabajo voluntario. El conocimiento de los proyectos de restauración en todas sus etapas, integra actores de diferentes niveles y conlleva a que los integrantes de las comunidades se apropien e involucren profundamente en los procesos de restauración, aportando, de ser necesario, gratuitamente innumerables horas de trabajo a cambio de la recuperación de los ecosistemas (Clewell y Aronson, 2005).

C9. Control de amenazas potenciales al ecosistema. Hace referencia al desarrollo de actividades ecológicas, técnicas y sociales que contribuyen en el control de acciones directa o indirectamente ejecutadas por intervención humana y favorecen la integridad de los ecosistemas involucrando su progresiva recuperación (SER, 2004; Vargas, 2007; González et al., 2008).

C10. Prioridad en la recuperación de la estructura, funcionamiento y servicios ecosistémicos. Las actividades de restauración son un esfuerzo práctico para recuperar de manera asistida o espontánea las dinámicas naturales que conducen al restablecimiento de algunas trayectorias posibles en los ecosistemas; no necesariamente en todos sus componentes, pero sí enfatizando en los que aseguran el mejoramiento de sus condiciones y la utilidad o provisión de servicios (SCDB, 2004; Vargas, 2007).

C11. Aplicación adecuada y clara de escalas temporales y espaciales en el proyecto. La SCDB (2004), Vargas (2007) y Orsi y Geneletti (2010), indican que los procesos de restauración ecológica requieren claridad en la determinación de tiempo necesario para el restablecimiento de las trayectorias sucesionales y componentes que se hayan proyectado (corto, mediano o largo plazo), así como identificación de la escala a la que se van a desarrollar (regional, local, parcela). 


\section{Criterios socio-ecológicos}

C12. Número de especies autóctonas o en peligro de extinción presentes para restauración. Las actividades de restauración en su mayoría requieren acción del hombre para garantizar en alguna medida el restablecimiento de las trayectorias ecológicas, para ello se evalúa la disponibilidad de germoplasma de especies endémicas existentes, que existieron o están en peligro de extinción evaluando trayectorias sucesionales, presencia en ecosistema de referencia, historia de vida, morfología, fenología, regeneración, adaptación e importancia para las comunidades (SER, 2004; Clewell y Aronson, 2005).

C13. Mecanismos de monitoreo para evaluar el proceso. La restauración ecológica requiere seguimiento continuo para evaluar los cambios que tiene el ecosistema con las actividades implementadas. El monitoreo garantiza el éxito en los procesos porque con sus resultados se obtiene información que sugiere la continuidad o modificación de las estrategias de restauración manejadas (Vargas, 2007; Ribeiro-Pinto, 2014).

Anexo 2. Percepciones de los actores por criterio.

\section{Percepciones}

C1. Participación en la gestión de la restauración e intercambio de saberes con las comunidades. El 100\% de actores locales conocieron el proceso, intercambiaron conocimientos y aunque consideran fundamental la participación en la formulación de proyectos no formaron parte de esta etapa. Todos los actores regionales conocieron el proyecto, pero solo un $78 \%$ considera que hubo intercambio de saberes; un 56\% afirma que la formulación la deben hacer las comunidades y reconocen que estas no estuvieron desde el origen del proyecto. De los actores nacionales, solo el $50 \%$ afirma haber conocido la iniciativa, el $75 \%$ manifiesta que hubo intercambio de saberes $y$, pese a aseverar que los proyectos deben ser formulados por un grupo interdisciplinario que incluya a las comunidades, reconocen que estas solo se incluyeron en la ejecución.

C2. Vinculación de diversos actores: dueños de tierra, Estado, comunidades y ONG. La totalidad de actores nacionales y locales $(100 \%)$ consideran importante la vinculación a procesos de restauración y, aunque no en la misma proporción $(89 \%)$, también así es percibida por los regionales. Todos los actores regionales y locales afirman que el proyecto vinculó a diferentes actores, destacando a niños que fueron capacitados y mujeres que en algunos casos participaron en construcción de cercas y plantación de árboles; mientras que el $25 \%$ de los nacionales considera que no fue así. Los tres tipos de actores en diferentes proporciones creen que es necesaria la sensibilización como estrategia para vincular las comunidades a los proyectos (local: $67 \%$, nacional: $50 \%$ y regional: $44 \%$ ) argumentando que en la mayoría de los casos hay desconocimiento de su importancia.

C3. Claridad conceptual y metodológica utilizada en el proyecto. Los actores locales, regionales y nacionales en un 89\%, 78\% y $50 \%$, respectivamente, afirman que hubo claridad y facilidad de comunicación para transmitir los conocimientos, pero todos los actores locales y el $22 \%$ de los regionales consideran que se requiere continuidad para fortalecer los conocimientos y aprendizaje; mientras los nacionales no tienen opinión sobre esto. Los actores locales que señalan falta de claridad lo hacen basados en la resistencia de algunos vecinos para recibir visitas de la Corporación y participar en el proyecto, porque consideraban que la entidad se apropiaría de sus predios. Los actores nacionales en un $50 \%$ consideran que no siempre las personas que atienden este tipo de iniciativas tienen claros los conceptos sobre restauración ecológica.

C4. Costo de uso del suelo. El $25 \%$ de actores nacionales, el $22 \%$ de regionales y el $17 \%$ de locales consideran que al ejecutar proyectos de restauración se reducen los ingresos económicos en los predios, principalmente si se dispone de áreas destinadas a desarrollar actividades de agricultura y ganadería que generan ingresos económicos. La totalidad de actores nacionales, regionales y locales coinciden en la importancia de crear incentivos económicos o eximir del pago de impuestos a propietarios de predios dedicados a proyectos de restauración o conservación. Los actores nacionales no tienen ninguna opinión sobre el incremento del valor de los predios al desarrollar proyectos de restauración; por el contrario, el $78 \%$ de los actores regionales y el $94 \%$ de los locales consideran que la ejecución del proyecto ha incrementado el valor de los predios porque se han construido mejoras en el caso de las cercas, se está favoreciendo el desarrollo de especies nativas maderables y no maderables que pueden ser aprovechadas a futuro de manera sostenible y se están mejorado las condiciones ambientales para favorecer la provisión de servicios ecosistémicos.

C5. Incorporación de prácticas de conservación, uso y aprovechamiento de ecosistemas buscando el equilibrio. Para todos los actores regionales, el $61 \%$ de los locales y el $50 \%$ de los nacionales el proceso de conservación y restauración debe iniciar con educación ambiental y posibilidades de realizar aprovechamiento sostenible de los recursos naturales existentes, debido a que en muchos casos se desconocen las prácticas adecuadas. El 50\% de actores locales y nacionales y el $44 \%$ de los regionales manifiestan que es importante generar alternativas productivas asociadas a la restauración como estrategia para brindar oportunidades a propietarios y comunidades locales; y de igual manera el $89 \%$ de los actores locales, el $44 \%$ de los regionales y el $25 \%$ de los nacionales consideran que las prácticas de conservación, uso y aprovechamiento sostenible se pueden incorporar a través de procesos de compensación, ordenamiento territorial y alternativas productivas y de mitigación con las que además de conservar e incentivar los procesos de restauración, se haga uso sostenible para el beneficio de las comunidades. 
C6. Contribución al mejoramiento de la calidad de vida. La totalidad de actores locales, el $75 \%$ de los nacionales y el $67 \%$ de los regionales coinciden en que el proyecto fue una oportunidad de empleo masculino, femenino y de ingresos adicionales, representados en venta de postes de madera, alimentación, transporte de insumos, materiales y alquiler de caballos. El $89 \%$ de los actores locales, el 75\% de los nacionales y el $67 \%$ de los regionales afirman que con el desarrollo del proyecto mejoraron los predios y condiciones económicas y ambientales que repercuten en la calidad de vida de los propietarios y comunidades locales. El 89\% de los actores locales, el 75\% de los nacionales y el 33\% de los regionales creen que la recuperación de los ecosistemas para provisión de servicios ambientales contribuye al mejoramiento de la calidad de vida.

C7. Intención común de cambiar los patrones que pueden estar influyendo en el deterioro. La totalidad de los actores nacionales y regionales y el $89 \%$ de los locales afirman que las principales causas de deterioro de los ecosistemas son el cambio de uso del suelo por deforestación y desarrollo de prácticas tradicionales como las quemas para "mejorar fertilidad en los suelos". El $72 \%$ de los actores locales, el $67 \%$ de los regionales y el $50 \%$ de los nacionales creen que hay intención de cambiar las prácticas tradicionales, pero es necesario implementar actividades de capacitación y tener la posibilidad de realizar aprovechamiento sostenible. Los actores locales (94\%), los nacionales (75\%) y los regionales (67\%) consideran que la principal razón para conservar está ligada a la disponibilidad del recurso hídrico.

C8. Aporte de trabajo voluntario. El $50 \%$ de actores nacionales y locales y el $22 \%$ de los regionales manifiestan que es importante el aporte de trabajo de las comunidades como una manera de contribuir al desarrollo de los proyectos para asegurar que existe compromiso de todas las partes. El $89 \%$ de los actores regionales, el $75 \%$ de los nacionales y el $50 \%$ de los locales afirman que la continuidad del proyecto debe estar a cargo de las comunidades asentadas en el área de influencia de desarrollo del proyecto, porque son ellas las directamente beneficiadas. El 33\% de los actores locales y el 25\% de los nacionales consideran fundamental adelantar gestiones a diferentes niveles para asegurar la continuidad del proyecto, los actores regionales no se pronuncian ante el tema.

C9. Control de amenazas potenciales al ecosistema. Todos los actores nacionales y regionales y un $89 \%$ de los locales identifican como principal amenaza la degradación y deforestación por talas, quemas, sobrepastoreo y ganadería extensiva y reconocen que a pesar de construir cercas el ganado sigue ingresando y los vecinos no están interesados en mejorar las condiciones de los ecosistemas. Los actores nacionales en un 50\%, los regionales en un $22 \%$ y los locales en un $11 \%$ consideran que para contrarrestar esta amenaza se puede hacer control con el fortalecimiento de la planificación predial que incentive el uso y aprovechamiento sostenible. Por su parte, la totalidad de actores regionales y el $72 \%$ de locales creen en la sensibilización ambiental e implementación de proyectos productivos como opción para fomentar la conservación y restauración, mientras que los actores nacionales no tienen ninguna opinión al respecto.

C10. Prioridad en la recuperación de la estructura, funcionamiento y servicios ecosistémicos. Los actores regionales y locales en su totalidad y el $75 \%$ de los nacionales manifiestan que hay especial interés en la provisión de servicios ecosistémicos y mejoramiento de la calidad de vida. Mientras el 94\% de los locales considera importante mejorar las condiciones ambientales para asegurar la disponibilidad del recurso hídrico, los regionales y nacionales no tienen ninguna opinión sobre esto. El total de actores locales, el $78 \%$ de los regionales y el $25 \%$ de los nacionales valoran como importante el favorecimiento de la conectividad para asegurar el hábitat de especies nativas.

C11. Aplicación adecuada y clara de escalas temporales y espaciales en el proyecto. Todos los actores regionales y locales y el $50 \%$ de los nacionales aseguran que las áreas seleccionadas fueron adecuadas y acordes a los lineamientos del proyecto. Un 33\% de los actores regionales, $25 \%$ de los nacionales y $6 \%$ de los locales creen que las áreas en que se realizó el proyecto fueron adecuadas, pero es importante ampliar este tipo de estrategias a otras áreas con necesidades similares. Respecto a la temporalidad del proyecto, el $94 \%$ de los actores locales, el $33 \%$ de los regionales y el $25 \%$ de los nacionales consideran que se presentaron demoras en las actividades de mantenimiento, situación que incrementó el porcentaje de mortalidad del material vegetal, la competencia de gramíneas y el deterioro de las cercas construidas.

C12. Número de especies autóctonas o en peligro de extinción presentes para restauración. 88\% de los actores regionales, $83 \%$ de los locales y $50 \%$ de los nacionales creen que las especies se seleccionaron de manera adecuada, de acuerdo a las condiciones de la región. La totalidad de actores locales, el $67 \%$ de los regionales y $25 \%$ de los nacionales consideran que en este tipo de proyectos es importante incluir especies amenazadas o extintas localmente que representen una opción de aprovechamiento forestal en el mediano y largo plazo. Por su parte, el 25\% de los actores nacionales manifiestan que es importante incluir especies nativas que aceleren los procesos de restauración, mientras los regionales y locales no tienen ninguna opinión al respecto.

C13. Mecanismos de monitoreo para evaluar el proceso. Los actores nacionales en un $75 \%$, los locales en un $33 \%$ y los regionales en un $11 \%$ consideran que la responsabilidad del monitoreo debe ser asumida por los actores regionales, locales y la academia, aun cuando este último actor solo empieza a tenerse en cuenta en esta etapa, pero no fue consultado en las etapas previas del proyecto. En el mismo sentido, la totalidad de actores nacionales y locales y el $77 \%$ de los regionales estiman conveniente que la verificación de efectividad de los procesos de restauración ecológica sea a través de parcelas de monitoreo y visitas periódicas como se hizo en el proyecto, porque permite verificar la efectividad del proceso o replantear las estrategias en caso de ser necesario. El $67 \%$ de actores regionales, el $50 \%$ de locales y el $25 \%$ de nacionales afirman que la responsabilidad del monitoreo debe ser de todos los actores para asegurar la continuidad de los procesos.

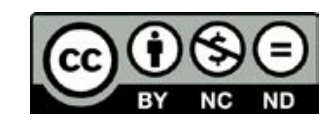

Colombia Forestal • ISSN 0120-0739 • e-ISSN 2256-201X • Bogotá-Colombia • Vol. 22 No. 1. Enero-Junio $2019 \bullet$ pp. 68-86.

[ 86 ] 